\title{
A THEOREM ON FOURIER-STIELTJES INTEGRALS
}

BY S. BOCHNER

1. Introduction. If $V(\alpha)$ is a (complex-valued) function of bounded variation in $-\infty<\alpha<\infty$,

$$
\int_{-\infty}^{\infty}|d V(\alpha)|=M<\infty
$$

then the function

$$
f(x)=\int_{-\infty}^{\infty} e^{i x \alpha} d V(\alpha)
$$

is a bounded continuous function in $-\infty<x<\infty$. We denote the class of these functions $f(x)$ by $\mathfrak{B}$. The distribution function $V(\alpha)$ which generates $f(x)$ is essentially unique* and we shall call the number $M$ the norm of $f(x)$.

A sub-class of $\mathfrak{B}$ is the class $\mathfrak{B}$ of those functions $f(x)$ whose distribution function is (real and) non-decreasing. The class of the latter functions coincides with the class of the so-called positive-definite functions for which I have recently given an independent characterization. $\dagger$ It is immediately seen that the functions of $\mathfrak{B}$ consist of all expressions

$$
f_{1}(x)-f_{2}(x)+i f_{3}(x)-i f_{4}(x)
$$

in which $f_{1}, f_{2}, f_{3}, f_{4}$ are any positive-definite functions. This indirect characterization of the class $\mathfrak{B}$ is of no interest. But we shall describe the class $\mathfrak{B}$ by an entirely different direct property, which is an imitation of a well known criterion due to F. Riesz.

THEOREM. In order that a bounded continuous function $f(x)$ be a function of $\mathfrak{B}$ with norm $\leqq M$ it is necessary and sufficient

* See S. Bochner, Vorlesungen über Fouriersche Integrale, Leipzig, 1932, p. $18 \mathrm{ff}$.

$\dagger$ Loc. cit. Compare also F. Riesz, Über Sätze von Stone und Bochner, Acta Szeged, vol. 6 (1933), pp. 184-198; and, for the case of several variables, S. Bochner, Monotone Funktionen, Stieltjessche Integrale und harmonische Analyse, Mathematische Annalen, vol. 108 (1933), pp. 378-410. 
that for any real numbers $x_{1}, x_{2}, \cdots, x_{m},(m=1,2, \cdots)$, and any numbers $c_{1}, c_{2}, \cdots, c_{m}$, we have

$$
\left|\sum_{\mu=1}^{m} c_{\mu} f\left(x_{\mu}\right)\right| \leqq M \cdot \sup _{-\infty<\alpha<\infty}\left|\sum_{\mu=1}^{m} c_{\mu} e^{i \alpha x_{\mu}}\right| .
$$

2. Remarks on the Theorem. The necessity of the condition is trivial; if we write

$$
L(\alpha ; c ; x)=\sum_{\mu=1}^{m} c_{\mu} e^{i \alpha x_{\mu}},
$$

we have

$$
\sum_{\mu=1}^{m} c_{\mu} f\left(x_{\mu}\right)=\int_{-\infty}^{\infty} L(\alpha ; c ; x) d V(\alpha),
$$

and (3) is an immediate consequence of (1). Before proving the sufficiency we shall insert a few remarks.

We consider the space $C$ of all bounded uniformly continuous functions $z(t)$ on a fixed finite or infinite interval $a<t<b$ with the usual norm

$$
\|z(t)\|=\sup _{0<t<b}|z(t)| .
$$

If the interval is finite, then, by a fundamental theorem of Riesz, every (continuous linear) functional $T z$ may be represented in the form

$$
T z=\int_{a}^{b} z(t) d V(t)
$$

where $V(t)$ is of bounded total variation in $(a, b)$. From this theorem of Riesz it is easy to deduce other theorems which in their wording are very similar to ours. This would suggest a deduction of our theorem from a generalization of the theorem of Riesz to the infinite interval $(-\infty, \infty)$. But the theorem of Riesz no longer holds in all its generality for infinite intervals, and our subsequent proof will proceed otherwise.

The failure of the general theorem of Riesz in the case of the interval $(-\infty, \infty)$ is illustrated by the following argument for which I am indebted to I. J. Schoenberg. Let $S$ denote the compact subspace of $C$ consisting of the almost periodic functions 
only, and let $T z$ be defined on $S ;^{*} T z$ is uniquely determined by the operator

$$
\phi(\lambda)=T e^{i \lambda t}, \quad(-\infty<\lambda<\infty) .
$$

If $T$ is representable in the form

$$
\int_{-\infty}^{\infty} z(t) d V(t)
$$

then

$$
\phi(\lambda)=\int_{-\infty}^{\infty} e^{i \lambda t} d V(t)
$$

and thus $\phi(\lambda)$ is continuous. But there are functionals $T z$ on $S$ whose operator is not continuous, and which, therefore, are not of Riesz's form; for instance, the functional

$$
\mathfrak{M}\{z\}=\lim _{T \rightarrow \infty} \frac{1}{2 T} \int_{-T}^{T} z(t) d t
$$

has the discontinuous operator $\phi(0)=1$, and $\phi(\lambda)=0$ for $\lambda \neq 0$.

Incidentally we observe that the operator $\phi(\lambda)$ satisfies the relation

$$
\left|\sum_{\mu=1}^{m} c_{\mu} \phi\left(\lambda_{\mu}\right)\right| \leqq M \cdot \sup _{-\infty<t<\infty}\left|\sum_{\mu=1}^{m} c_{\mu} e^{i t \lambda_{\mu}}\right|,
$$

where $M$ is the norm of the given functional $T$, and conversely, if a (continuous or discontinuous) function $\phi(\lambda)$ satisfies this relation, then there exists a functional $T$ with this function as operator. From our theorem we may therefore draw the following conclusion. In order that a functional $T z$ in the space of the uniformly continuous almost periodic functions be representable in the form of Riesz, it is necessary and sufficient that its operator $\phi(\lambda)$ be continuous.

Finally we shall make a remark about the set of points $x$ for which the condition (3) has to be satisfied. In the wording of the theorem, we have required that this condition shall be fulfilled for any real points $x_{\mu}$. But our subsequent proof will show

* We recall that by a theorem of Banach and Hahn, $T z$ may be extended over $C$. 
that it would be sufficient to restrict ourselves to the rational points, say. But we want to point out that it would not do to restrict the points $x_{\mu}$ to any set which is everywhere dense on the real axis. For instance, if the points $x_{1}, \cdots, x_{m}$ are linearly independent, then

$$
\sup \left|\sum_{\mu=1}^{m} c_{\mu} e^{i \alpha x_{\mu}}\right|=\sum_{\mu=1}^{m}\left|c_{\mu}\right|
$$

and the condition (3) is certainly fulfilled if $|f(x)| \leqq M$. But it is not true that any bounded continuous function $f(x)$ belongs to the class $\mathfrak{B}$.

3. Proof of the Theorem. For the proof of the sufficiency of our condition we need two lemmas.

Lemma 1. If a sequence of functions $f_{n}(x) \subset \mathfrak{B}$, with bounded norms,

$$
M_{n} \leqq \bar{M},
$$

is uniformly convergent on every finite interval, then the limit function $f_{0}(x)$ also belongs to $\mathfrak{B}$, and its norm is also $\leqq \bar{M}$.

Proof. Assuming that $V_{n}(-\infty)=0$, and taking a suitable subsequence of $f_{n}(x)$, we can add the hypothesis that the sequence $V_{n}(\alpha)$ converges almost everywhere to a function $V_{0}(\alpha)$ for which

$$
\int_{-\infty}^{\infty}\left|d V_{0}(\alpha)\right| \leqq \bar{M}
$$

And now it follows, as in the case of positive-definite functions $f_{n}(x)$, that the limit function $f_{0}(x)$ has the distribution function $V_{0}(\alpha),{ }^{*}$ which proves the lemma.

Lemma 2. If $f(x)$ is a bounded continuous function satisfying condition (3), then, for each $t>0$, the functions

$$
\begin{aligned}
& g_{t}(x)=\frac{1}{t} \int_{0}^{t} f(x+\tau) d \tau, \\
& h_{t}(x)=e^{-x^{2} t} f(x),
\end{aligned}
$$

also satisfy this condition, and for the same constant $M$.

Proof. The assertion about $g_{t}(x)$ follows immediately from

\footnotetext{
* Bochner, Vorlesungen über Fouriersche Integrale, pp. 71-72.
} 


$$
\sup _{\alpha}\left|\sum_{\mu=1}^{m} c_{\mu} e^{i \alpha\left(x_{\mu}+1\right)}\right|=\sup _{\alpha}\left|\sum_{\mu=1}^{m} c_{\mu} e^{i \alpha x_{\mu}}\right|,
$$

and

$$
\left|\sum_{\mu=1}^{m} c_{\mu} g_{t}\left(x_{\mu}\right)\right| \leqq \frac{1}{t} \int_{0}^{t}\left|\sum_{\mu=1}^{m} c_{\mu} f\left(x_{\mu}+\tau\right)\right| d \tau .
$$

For fixed $t>0$, we have

$$
e^{-x^{2} t}=\int_{-\infty}^{\infty} F(\beta) e^{-i \beta x} d \beta,
$$

where

$$
F(\beta)=2\left(\frac{t}{\pi}\right)^{1 / 2} e^{-t^{2} \beta / 4}
$$

is a positive function. Hence

$$
\begin{gathered}
\left|\sum_{\mu=1}^{m} c_{\mu} h_{t}\left(x_{\mu}\right)\right|=\left|\sum_{\mu=1}^{m} c_{\mu} e^{-x_{\mu}{ }^{2} t} f\left(x_{\mu}\right)\right| \leqq M \cdot \sup _{\alpha}\left|\sum_{\mu=1}^{m} c_{\mu} e^{-x_{\mu}{ }^{2} t+i \alpha x_{\mu}}\right| \\
\leqq M \cdot \sup _{\alpha} \int_{-\infty}^{\infty} F(\beta)|L(\alpha-\beta ; c ; x)| d \beta \\
\leqq M \cdot \sup _{\alpha}|L(\alpha ; c ; x)| \cdot \int_{-\infty}^{\infty} F(\beta) d \beta=M \cdot \sup _{\alpha}|L(\alpha ; c ; x)| .
\end{gathered}
$$

Now let $f(x)$ be a bounded continuous function satisfying condition (3). We form, for $t>0$, the functions

$$
\begin{gathered}
p_{t}(x)=e^{-x^{2} t} f(x), \quad q_{t}(x)=\frac{1}{t} \int_{0}^{t} p_{t}(x+\tau) d \tau \\
f_{t}(x)=\frac{1}{t} \int_{0}^{t} q_{t}(x+\tau) d \tau
\end{gathered}
$$

By Lemma 2, we have

$$
\left|\sum_{\mu=1}^{m} c_{\mu} f_{t}\left(x_{\mu}\right)\right| \leqq M \cdot \sup _{\alpha}|L(\alpha ; c ; x)| .
$$

But it is easily seen that $f_{t}(x)$ may be written in the form 


$$
f_{t}(x)=\int_{-\infty}^{\infty} e^{i x \alpha} \phi_{t}(\alpha) d \alpha
$$

with a transform $\phi_{t}(\alpha)$ which is continuous and absolutely integrable in $-\infty<\alpha<\infty$. Substituting (7) in (6), we get

$$
\left|\int_{-\infty}^{\infty} L(\alpha ; c ; x) \phi_{t}(\alpha) d \alpha\right| \leqq M \cdot \sup _{\alpha}|L(\alpha ; c ; x)| .
$$

Every periodic continuous function $P(\alpha)$ can be uniformly approximated by expressions (4); therefore

$$
\left|\int_{-\infty}^{\infty} P(\alpha) \phi_{t}(\alpha) d \alpha\right| \leqq M \cdot \sup _{\alpha}|P(\alpha)| .
$$

To fixed $t>0$ and any $\epsilon>0$ we can assign a finite number of finite closed intervals; let their sum be denoted by $\mathfrak{A}$, such that $\left|\phi_{t}(\alpha)\right|$ is essentially positive on $\mathfrak{A}$, and that the integral on the complementary set of $\left|\phi_{t}(\alpha)\right|$ is $<\epsilon$. We can construct a continuous periodic function $P(\alpha)$, with a suitable period, whose absolute value is $\leqq 1$, and which, on the set $\mathfrak{A}$, has the value

Hence

$$
\frac{\phi_{t}(\alpha)}{\left|\phi_{t}(\alpha)\right|} \text {. }
$$

$$
\begin{aligned}
\int_{-\infty}^{\infty}\left|\phi_{t}(\alpha)\right| d \alpha & \leqq \epsilon+\int_{\mathfrak{A}}\left|\phi_{t}(\alpha)\right| d \alpha=\epsilon+\int_{\mathfrak{A}} P(\alpha) \phi_{t}(\alpha) d \alpha \\
& \leqq \epsilon+\epsilon+\left|\int_{-\infty}^{\infty} P(\alpha) \phi_{t}(\alpha) d \alpha\right| \leqq 2 \epsilon+M \cdot 1
\end{aligned}
$$

and, if $\epsilon \rightarrow 0$,

Putting

$$
\int_{-\infty}^{\infty}\left|\phi_{t}(\alpha)\right| d \alpha \leqq M
$$

$$
V_{t}(\alpha)=\int_{0}^{\alpha} \phi_{t}(\beta) d \beta
$$

we find that $f_{t}(x)$ is a function of $\mathfrak{B}$, with a norm $\leqq M$. For $t \rightarrow 0, f_{t}(x)$ converges to $f(x)$ uniformly on every finite interval. By Lemma $1, f(x)$ is a function of $\mathfrak{B}$ and its norm is $\leqq M$.

Princeton University 